\title{
Virus-Cell Relation in Rat Polyoma Tumours
}

\author{
By M. VANDEPUTTE AND P. DE SOMER \\ Rega Institute, University of Louvain, Belgium
}

(Received 27 November 1961)

\begin{abstract}
SUMMARY
Experiments on rat tumours induced by polyoma virus indicate that, although no virus was directly demonstrable in these tumours, at least by the technique used, some of the tumours when set up as tissue cultures were still capable of synthesizing polyoma virus. This faculty disappeared, however, in most instances after transplantation in vivo; virus recovery by tissue culture procedures after passage in rats was exceptional. Furthermore, if one draws a parallel between the virus/cell relationship in rat polyoma tumours and the lysogenic system in bacteria, one finds that both phenomena though not exactly similar have many points in common.
\end{abstract}

\section{INTRODUCTION}

Most of the studies on the virus/cell relationship in tumours induced by polyoma virus have been made on primary and transplantable tumours in hamsters (Habel \& Silverberg, 1960) and in mice (Sachs \& Fogel, 1960; Winocour \& Sachs, 1961). These studies suggested that differences in the virus/cell relationship might depend on the experimental animal chosen. For instance, whereas mouse tissue was shown to have not only a non-lytic but also a strong lytic response to polyoma virus in vivo and in vitro (Stanton, Stewart, Eddy \& Blackwell, 1959; Sachs \& Fogel, 1960), hamster tissue upon infection reacted predominantly by a non-lytic response with rapid tumour formation (Vogt \& Dulbecco, 1960; Stoker \& MacPherson, 1961; Roizman \& Roane, 1960).

In the present work we studied the virus/cell relationship in polyoma tumours induced in our $\mathbf{R}$ inbred strain of rats. Previous experiments (Vandeputte, 1961) showed that no polyoma virus could be recovered from meningeal sarcomas induced by this virus in our rat strain. To determine whether this finding applied to other rat tumours, we analysed different primary and transplantable rat polyoma tumours both directly and in vitro for the presence of the virus or its antigen, using the fluorescent antibody technique and tissue culture procedures. We also studied the behaviour of these tumours when transplanted and when challenged by polyoma virus in vitro.

\section{METHODS}

Virus stock. The polyoma strain used in our experiments was obtained from Dr C. Dawe (National Institutes of Health, Bethesda, Md., U.S.A.). The virus was grown on mouse embryo fibroblast cultures in our laboratory; the stock virus used had a titre of $2 \times 10^{6}$ Tissue Culture Infectious Dosis 50/ml. (TCID 50/ml.).

Primary and transplanted tumours in the rat. Within $24 \mathrm{hr}$. after birth, rats of the Albino R strain (obtained from Prof. Mühlbock, Amsterdam) were inoculated intra- 
cerebrally or subcutaneously with $10^{5}$ TCID 50 doses of polyoma virus. The rats which developed tumours were bled-out and after heating the sera to $56^{\circ}$ for $30 \mathrm{~min}$. the haemagglutination-inhibition antibody titre was determined, using 10 haemagglutinating units (Rowe, Hartley, Estes \& Huebner, 1959). Each tumour was removed aseptically and part of it frozen immediately either for cryostat frozen sections or for fluorocarbon extraction. Another part was trypsinized and set up as a tissue culture. When a tumour was very large, a fragment was also minced with scissors and grafted subcutaneously into isologous adult rats. Meningeal sarcomas and liposarcomas were not trypsinized; they were used only for transplantation or for fluorescent antibody studies and fluorocarbon extraction. Primary tumours that grew after grafting into isologous rats were serially transplanted and fragments from them were trypsinized for fluorescent antibody studies and polyoma virus isolation in tissue culture.

Tissue culture preparation of rat polyoma tumours. A portion of the primary tumour was trypsinized, and the cell suspension obtained after washing three times was put into tissue culture. For fluorescent antibody studies Leighton tubes were inoculated with $10^{5}$ cells, and for virus isolation Petri dishes $(60 \mathrm{~mm}$ diam.) were inoculated with $10^{6}$ cells and kept in a humidified incubator supplied with a constant flow of $5 \% \mathrm{CO}_{2}$ in air. In all tissue culture experiments Eagle's medium with $10 \%$ $(\mathrm{v} / \mathrm{v})$ calf serum was used. After $24 \mathrm{hr}$. and after 4, 6, 8 and 10 days tissue cultures were removed to see whether viral antigen could be detected by the fluorescent antibody technique and whether virus could be isolated from the centrifuged supernatant medium, and from the remaining medium together with the cells after these had been frozen and thawed three times.

One or two Petri dish cultures were kept to establish a continuous line of the tumour cells. After two to three in vitro passages the cells from these Petri dishes were inoculated into young (about 1-3 days old) and adult isologous rats. The subcutaneous tumours obtained by direct grafting of the primary tumour as mentioned above, or by injecting subcutaneously cells of the 2nd or 3rd tissue culture passages from these primary tumours, were aseptically removed after bleeding-out the rat and were retransplanted (2nd passage Tables 1 and 2). A portion of each tumour was trypsinized and put in tissue culture to test for the presence of viral antigen and infectious polyoma virus.

Determination of the presence of infectious polyoma virus. Frozen pieces of primary rat polyoma tumours were made up as a $10 \%$ suspension by grinding in a mortar in phosphate-buffered saline $\left(\mathrm{NaCl} 9 \mathrm{~g} .+\mathrm{NaH}_{2} \mathrm{PO}_{4} \cdot \mathrm{H}_{2} \mathrm{O} 0.519 \mathrm{~g} .+\mathrm{Na}_{2} \mathrm{HPO}_{4} \cdot 2 \mathrm{H}_{2} \mathrm{O}\right.$ $1.111 \mathrm{~g} . / 1$.) and treated twice in the cold by fluorocarbon (Freon 113) in a 'Polytron' Omnimix (M. Wullimann, Selzach, Switzerland) at high speed (10,000 rev./min.). For polyoma virus isolation $0 \cdot 1 \mathrm{ml}$. of the aqueous phase was inoculated into mouse embryo tissue culture. The tissue culture samples from primary and transplanted rat polyoma tumours were also inoculated on mouse embryo tissue culture (0-1 ml. of each sample in two tissue culture tubes). These inoculated tubes were kept for 12 days and examined every 2 days for the appearance of cytopathic effect. After 12 days, the presence of haemagglutinating activity in the medium was determined. Material from tissue culture tubes which showed no cytopathic effect or haemagglutinating activity was inoculated into new mouse embryo tissue culture tubes and the corresponding initial tumour extracts were inoculated intraperitoneally $(0 \cdot 2 \mathrm{ml}$.) 
into adult mice. These were bled after 3 and 6 weeks for the titration of haemagglutination-inhibition antibodies, 10 haemagglutinating units being used (Rowe's MAP test, 1959).

Fluorescent antibody studies. Frozen tissue blocks were sectioned at an indicated thickness of $6 \mu$ in a $-20^{\circ}$ microtome cryostat. These sections were air-dried at room temperature for $10 \mathrm{~min}$. and then fixed in anhydrous acetone for $15 \mathrm{~min}$. After air-drying and repeated washing in phosphate-buffered saline, antipolyoma chicken serum diluted 1/5 was applied on the sections which were then incubated for $30 \mathrm{~min}$. at $37^{\circ}$ in a moistened Petri dish. This serum had previously been absorbed three times with mouse fibroblasts and had a titre of $1 / 2048$ as determined by the haemagglutination-inhibition test. After incubation, the slides were washed three times in phosphate-buffered saline and re-incubated at $37^{\circ}$ for $30 \mathrm{~min}$. with fluoresceinlabelled antichicken goat serum (Microbiological Associates. Inc. Bethesda, Md., U.S.A.), which had previously been absorbed twice with acetone-dried mouse liver powder. As counterstain, we used Lissamine Rhodamine RB-200 conjugated with bovine albumin (Microbiological Associates). Before being mounted in buffered glycerol, the slides were washed in three changes of phosphate-buffered saline.

To test the specificity of the action, slides were treated in parallel with normal chicken serum before the application of fluorescein-conjugated antichick serum and with the fluorescein-conjugated serum alone. Mouse embryo fibroblasts not infected with polyoma virus were also used as a control to detect the presence of any nonspecific fluorescence. The fluorescence procedure on tissue culture was similar, except that before air-drying and fixation in anhydrous acetone the slides were washed three times with phosphate-buffered saline.

The optical system consisted of the Zeiss fluorescence microscope equipped with a high pressure mercury lamp HBO 200; the BG 12 (3 mm. $\times 5$ mm.) exciter filters and the OG5 and OG4 barrier filters were used.

\section{RESULTS}

\section{Primary tumours of the rat}

Primary tumours were studied for the presence of viral antigen by the indirect fluorescent antibody technique on frozen cryostat sections. As shown in Table 1, no polyoma antigen could be demonstrated in the nine tumours tested (Pl. 1, fig. 1). Tumour extracts prepared in phosphate-buffered saline, whether treated or untreated with fluorocarbon, were negative for the presence of polyoma virus even after a blind passage on mouse embryo tissue culture (Table 2). The fluorocarbon technique was used to separate the antibodies from any virus which might be present in the extract (Hummeler \& Ketler, 1958). Samples of the same tumour extracts injected intraperitoneally in adult mice gave negative MAP tests. These data indicated that the infectious polyoma virus and its antigen were no longer detectable in the tumours or their extracts, at least by the techniques used.

However, when portions of those same tumours, found negative by direct examination as above for infectious virus or viral antigen, were put up as tissue cultures, the synthesis of polyoma virus was now detected in four of ten tumours (Table 1). By the fluorescent antibody technique, we found the viral antigen to be localized mostly in the nuclei (Pl. 1, figs. 2, 3) and only occasionally in the cytoplasm 
(Pl. 2, fig. 4). Synthesis of the antigen reached a peak from the 4th to the 6 th days and then decreased to disappear sometimes after 9 days (Table 1 ). This implies that the cells in which viral antigen was synthesized had died and that there was no re-infection of other cells by any released polyoma virus. From all the tumours set up as tissue cultures and yielding viral antigen as shown by the fluorescent antibody technique, infectious virus was isolated on mouse embryo tissue culture.

The appearance of viral antigen preceded the release of infectious virus in the supernatant medium (Table $2, \mathrm{RKS}_{1}, \mathbf{R K S}_{4}$ ) but probably not the detection of intracellular infectious virus. Those tumours set up as tissue cultures which did not

Table 1. Fluorescent cells in rat tumours (\%); indirect method

\begin{tabular}{|c|c|c|c|c|c|c|}
\hline \multirow{2}{*}{$\begin{array}{c}\text { Type } \\
\text { of rat } \\
\text { tumour* }\end{array}$} & \multirow{2}{*}{$\begin{array}{c}\text { Frozen } \\
\text { cryostat } \\
\text { sections }\end{array}$} & \multicolumn{5}{|c|}{ Primary tissue culture } \\
\hline & & $24 \mathrm{hr}$. & Day 4 & Day 6 & Day 8 & Day 10 \\
\hline $\mathbf{R K S}_{\mathbf{1}}$ & nt & $5 / 1 \ddagger$ & $7 / 2$ & $6 / 2$ & $3 / 0$ & $\mathbf{1} / \mathbf{1}$ \\
\hline $\mathbf{R K S}_{2}$ & - & $5 / 0$ & $4 / 1$ & $1 / 0$ & $0 / 1$ & $\mathbf{0} / \mathbf{0}$ \\
\hline $\mathbf{R K S}_{3}$ & - & - & - & - & - & - \\
\hline $\mathbf{R K S}_{4}$ & - & $3 / 0$ & $5 / 4$ & $\mathbf{1} / \mathbf{0}$ & nt & $1 / 0$ \\
\hline RKS $_{5}$ & - & - & - & - & nt & - \\
\hline RKS $_{8}$ & nt & - & - & - & - & - \\
\hline $\mathbf{R K S}_{\mathbf{7}}$ & nt & - & - & nt & - & - \\
\hline $\mathrm{RFS}_{1}$ & - & $4 / 1$ & $6 / 3$ & $1 / 1$ & nt & $0 / 0$ \\
\hline $\mathrm{RFS}_{2}$ & - & - & nt & nt & - & - \\
\hline $\mathbf{R F S}_{3}$ & - & - & - & nt & - & - \\
\hline $\mathbf{R I S}_{1}$ & - & nt & nt & nt & nt & nt \\
\hline $\mathbf{R M S}_{1}$ & - & nt & nt & nt & nt & nt \\
\hline
\end{tabular}

* RKS = rat kidney sarcoma; RFS = rat subcutaneous fibrosarcoma; RLS = rat liposarcoma; RMS = rat meningeal sarcomatosis.

$\dagger \mathbf{n t}=$ not tested; $-=$ negative.

$\ddagger$ First number = nuclear fluorescence; second number $=$ cytoplasmic fluorescence (determined on 100 to 200 cells).

Table 2. Virus isolation from rat tumour cells Phos-

Fluoro- phate-

Primary tissue culture

Tissue culture after passage in rats

Rat carbon buffer
tumours* extract $\dagger$ extract

$\overbrace{24 \mathrm{hr} . \quad \text { Day } 4}$ Day 6 Day 8 Day 10
$\overbrace{\text { 1st pass. HIA } \ddagger \text { 2nd pass. HIA } \ddagger}^{\text {2nd }}$

\begin{tabular}{|c|c|c|c|c|c|c|c|c|c|c|c|}
\hline $\mathbf{R K S}_{1}$ & - & nt & $+1-8$ & $+1+$ & $+1+$ & nt & nt & nt & - & - & - \\
\hline $\mathbf{R K S}_{\mathbf{2}}$ & nt & nt & $+1+$ & $+1+$ & nt & $+1+$ & nt & - & - & - & - \\
\hline $\mathbf{R K S}_{3}$ & nt & nt & - & - & nt & - & - & - & - & - & - \\
\hline $\mathrm{RKS}_{4}$ & nt & - & $+1-$ & $+1+$ & nt & $+1+$ & $+1+$ & - & - & $+1--1$ & - \\
\hline $\mathbf{R K S}_{5}$ & nt & nt & - & - & - & - & - & - & - & - & - \\
\hline $\mathbf{R K S}_{6}$ & - & nt & - & nt & - & - & - & nt & nt & nt & nt \\
\hline $\mathbf{R K S}_{7}$ & - & nt & - & - & - & - & - & nt & nt & nt & nt \\
\hline $\mathbf{R K S}_{8}$ & - & nt & nt & nt & nt & nt & nt & nt & nt & nt & nt \\
\hline $\mathbf{R F S}_{1}$ & - & nt & nt & $+1+$ & $+1+$ & $+1+$ & $+1+$ & - & - & - & - \\
\hline $\mathbf{R F S}_{2}$ & nt & - & - & - & - & nt & - & nt & nt & nt & nt \\
\hline $\mathrm{RFS}_{3}$ & nt & - & - & - & nt & - & - & nt & nt & nt & nt \\
\hline RFS $_{4}$ & - & - & nt & nt & nt & nt & nt & nt & nt & nt & nt \\
\hline
\end{tabular}

* See Table 1 for types.

$\dagger$ nt $=$ not tested; $-=$ negative.

$\ddagger$ Haemagglutination-inhibition antibody (HIA) titre in the tumour-bearing rats (less than $1 / 32$ is considered negative).

$\S$ First sign $=$ intracellular virus; second sign = virus in supernatant medium.

II One tumour out of three set up as tissue cultures released polyoma virus. 
then yield for infectious polyoma virus did not show any specific fluorescence which could indicate the synthesis or presence of incomplete virus (Pl. 2, fig. 5). Sera from mice inoculated with samples of these negative tissue cultures gave negative MAP tests.

\section{Transplantable rat polyoma tumours}

An attempt to transplant fragments of primary tumours minced with scissors by subcutaneous grafting into isologous rats was not very successful. Indeed, two meningeal sarcomas, two kidney sarcomas and one subcutaneous fibrosarcoma failed to grow after being grafted into isologous hosts. Only two kidney sarcomas $\left(\mathbf{R K S}_{7}, \mathbf{R K S}_{8}\right)$ could be serially transplanted. After one and three in vivo passages, portions of these transplantable tumours were put up as tissue cultures. Both failed to release infectious polyoma virus and neither contained viral antigen. The rats bearing these transplantable tumours had consistently negative HA-inhibiting antibody titres. The transplantation into rats of primary tumours after one or two passages in tissue cultures was more successful (Tables 1, 2). These tumours grew very well in all the inoculated young rats (1-3 days old) and gave tumours in $60-80 \%$ of the adults. The young and adult rats which developed tumours at the site of inoculation of these tissue cultures (1st passage) and those bearing transplants of these Ist passage tumours (2nd passage, see Table 2) never developed haemagglutination-inhibiting antibodies against polyoma virus.

All the first passage tumours when put up as tissue cultures were found negative for the presence of viral antigen as tested by the fluorescent antibody technique and for infectious polyoma virus (Table 2 ).

One tumour from the second in vivo passage released virus in tissue culture $\left(\mathbf{R K S}_{4}\right)$. Curiously, two tumours that arose from the same inoculum (1st passage tumour) in two other rats from the same nest and grafted at the same time were found negative for polyoma virus when put up as tissue cultures. This seems to indicate that although there is generally no virus production after transplantation in vivo of polyoma-induced tumours of the rat, exceptionally some tumour lines are still capable of synthesizing and releasing virus when set up as tissue cultures. The reasons for this particular behaviour are still unknown. The difficulties encountered in the transplantation experiments of primary polyoma tumours into isologous hosts could be due either to factors depending on the host, such as genetic differences, or to factors determined by the tumour itself, such as the acquisition of new antigens, nutritional deficiency, etc. Our Albino $\mathbf{R}$ rat strain seems to be genetically homogeneous, at least for the histocompatibility antigens. This was verified by skin transplantation between animals chosen at random and by transplantation of one spontaneous mammary tumour and two methylcholanthrene-induced fibrosarcomas. This indicates that polyoma tumours in rats behave on transplantation like some polyoma tumours in the mouse (Dawe, 1960). Primary hamster polyoma tumours on the other hand seem to be easily transplantable, as shown by Habel \& Silverberg (1960). Our data suggest that the difficulties encountered in transplanting primary rat tumours are determined by factors inherent in the tumour rather than in the host. These factors are slightly depressed by passage in tissue culture and are overcome by the use of newborn rats for transplantation, as previously described. Further studies are necessary, however, to determine the exact nature of this phenomenon. 


\section{Susceptibility of rat polyoma tumours to challenge infection with polyoma virus}

Tissue cultures made from three primary kidney sarcomas and one subcutaneous fibrosarcoma were challenged with $2 \times 10^{5}$ TCID 50 doses of polyoma virus. Two of these lines $\left(\mathbf{R K S}_{1}, \mathbf{R K S}_{2}\right)$ were initially positive for virus production in tissue culture. In all cases, these tissue culture lines were found resistant to superinfection by polyoma virus, and no cytopathic effect or virus production was observed during an observation period of 12 days. For challenge, the same strain of polyoma virus as that which induced the tumours was used. In parallel with these experiments, we examined also the susceptibility to polyoma virus of a normal rat kidney continuous line (RN cells) and a continuous tissue culture line established from a carcinogeninduced mammary tumour (DE 40 cells); these two lines were also found resistant to infection by polyoma virus. This indicates that the resistance of tissue culture lines established from rat polyoma tumours to challenge infection by this virus is not a specific character of the tumours. The polyoma tissue culture lines, like the $\mathrm{RN}$ and $\mathrm{DE} 40$ cells, were susceptible to herpes and vaccinia virus.

\section{DISCUSSION}

Sachs \& Fogel (1960) showed that rat polyoma tumours, when put in tissue culture, synthesized infectious virus and viral antigen. Although our studies point in the same direction, we did not find this to be a general phenomenon. Putting a rat polyoma tumour found negative by direct examination for the presence of virus in tissue culture seems to induce only in some instances the synthesis of polyoma virus. The other tumours, which did not release infectious virus in tissue culture, likewise appeared to contain no viral antigen when analysed by the fluorescent antibody technique and the MAP test. No evidence was found for the existence of an incomplete virus in our rat tumour lines as described by Sachs \& Fogel (1960) for certain mouse and hamster polyoma tumours. In our tumours, which were positive in tissue culture for the presence of virus, we found mostly a nuclear and only occasionally a cytoplasmic localization of the viral antigen. These results differ from those reported by Sachs \& Fogel (1960), who always located the viral antigen in the cytoplasm of his tumour cells. We do not know yet whether this difference may result from the greater sensitivity of the indirect method as compared with the direct fluorescent antibody method used by Sachs.

The induction by tissue culture of virus production in rat tumours found negative for polyoma virus on direct examination, the rate of synthesis of the virus as shown by the fluorescent antibody technique and the disappearance of the virus on transplantation with occasional recovery in tissue culture, suggest a virus/cell relationship close to a lysogenic system, as was indicated by Winocour \& Sachs (1961) for polyoma tumours in the mouse. The non-susceptibility of the polyoma tumour lines to superinfection by the same virus and their susceptibility to unrelated viruses (herpes and vaccinia virus).might be considered as another point of similarity if it had been specific for the rat tissue culture lines originated from polyoma tumours only. Also the possible acquisition of a new cellular antigen, as suggested by our preliminary transplantation experiments, has its equivalent in the bacterial lysogenic system 


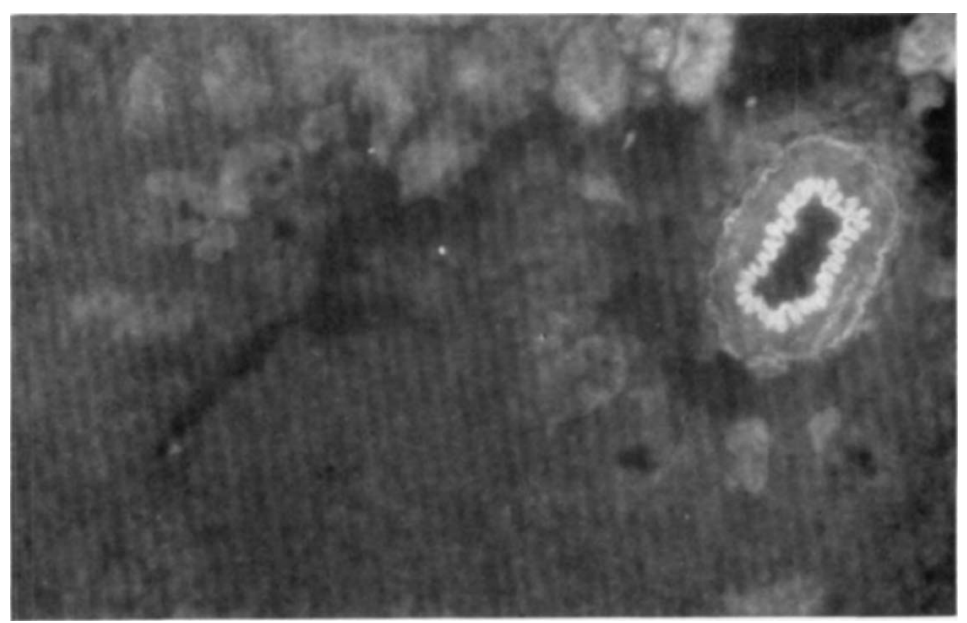

Fig. 1

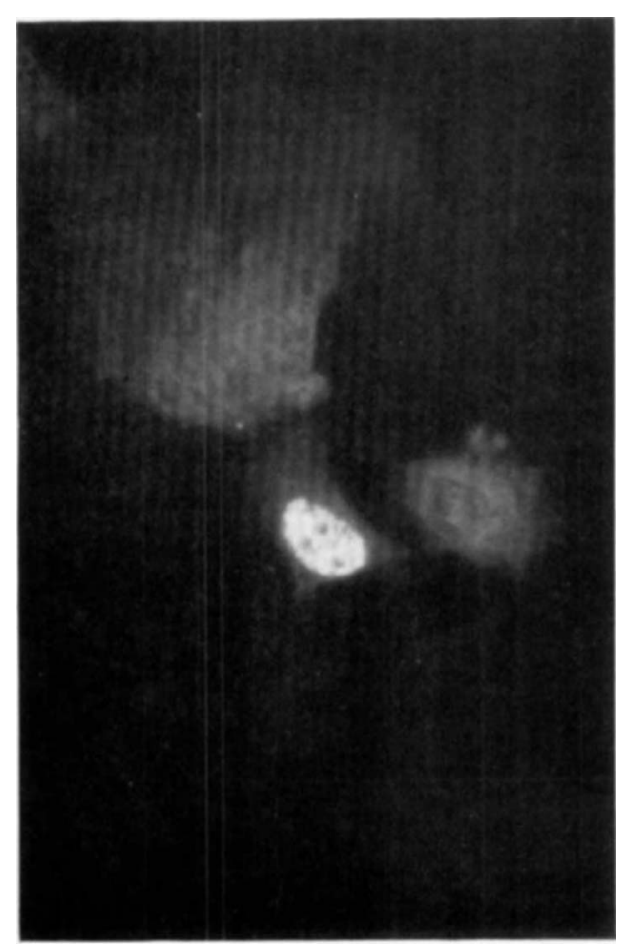

Fig. 2

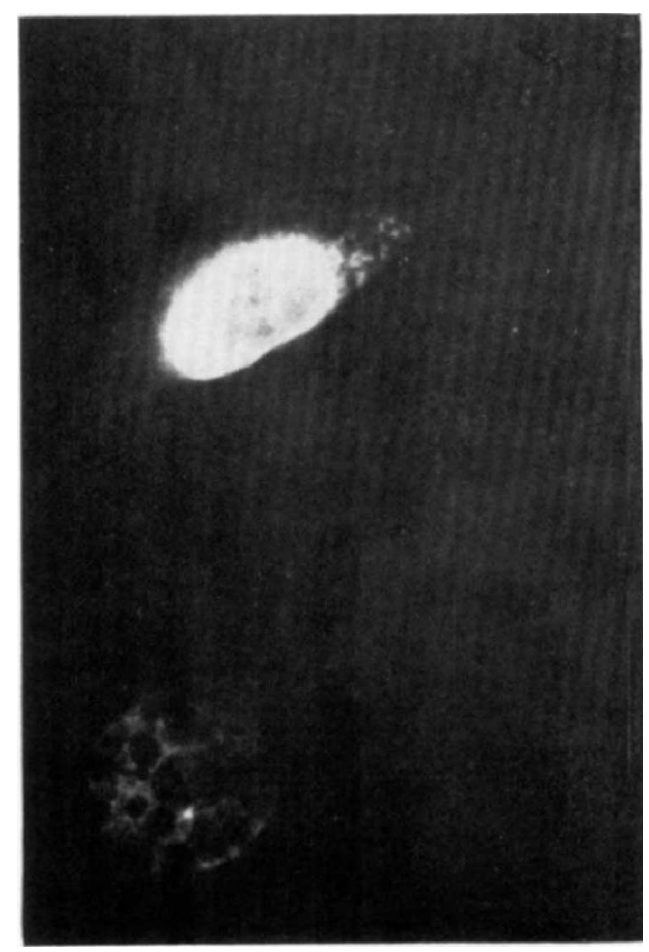

Fig. 3 


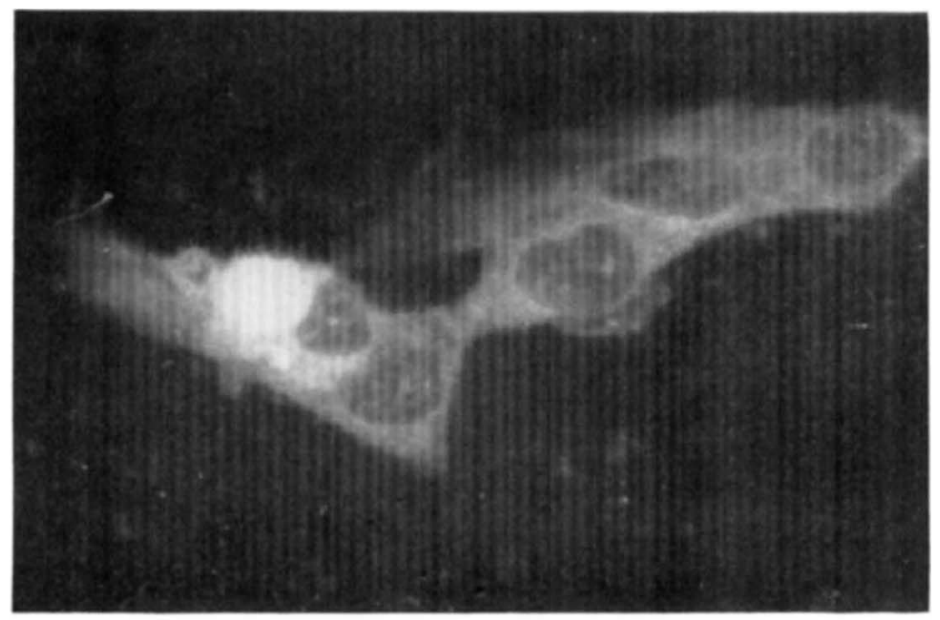

Fig. 4

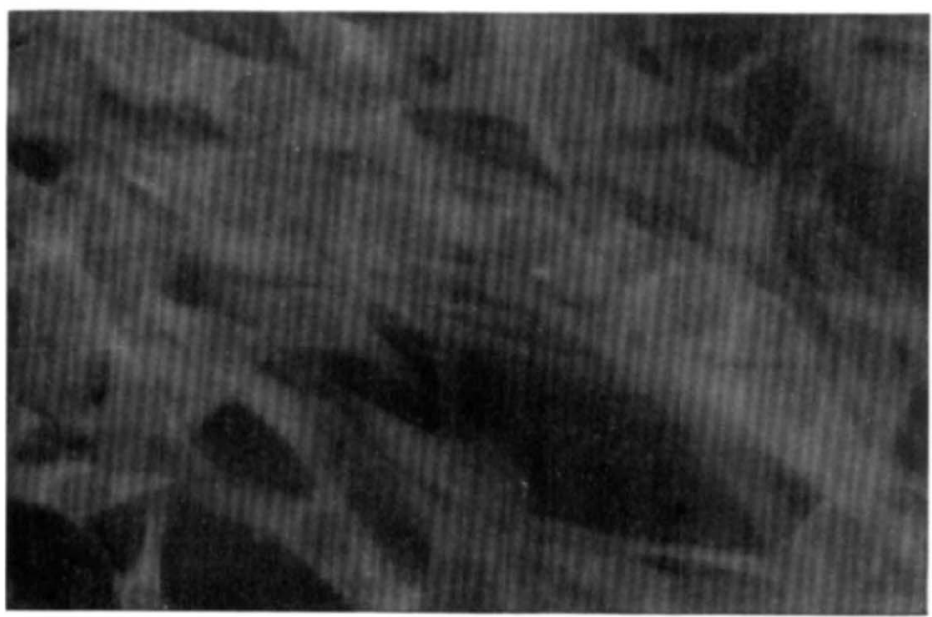

Fig. 5 
(Jacob \& Wollman, 1959); further studies will indicate whether our findings are analogous to those described by Sjögren, Hellström \& Klein (1961), in mouse polyoma tumours.

Part of this work was presented at the Meeting of the Society for General Microbiology held in Oxford (28-30 September 1961).

\section{REFERENCES}

Dawe, C. J. (1960). Cell sensitivity and specificity of response to polyoma virus. Nat. Cancer Inst. Monograph, no. 4, 67.

Habel, K. \& Silverberg, R. J. (1960). Relationship of polyoma virus and tumor in vivo. Virology, 12, 463.

Hummeler, K. \& KetheR, A. (1958). Dissociation of poliomyelitis virus from neutralizing antibody. Virology, 6, 297.

JACOB, F. \& Wolman, E. L. (1959). Lysogeny-The Viruses, 2, 1st ed. New York: Academic Press Inc.

Rolzman, B. \& Roane, P. (1960). Studies of polyoma virus. J. Immunol. 85, 429.

Rowe, W. P., Hartley, J. W., Estes, J. D. \& Huebner, R. J. (1959). Studies of mouse polyoma virus infection. I Procedures for quantitation and detection of virus. J. exp. Med. 109, 879.

SACHS, L. \& Fogel, M. (1960). Polyoma virus synthesis as measured by the fluorescent antibody technique. Virology, 11, 722.

Sjögren, H. O., Hellström, I. \& Krein, G. (1961). Transplantation of polyoma virusinduced tumours in mice. Cancer Res. 21, 329.

Stanton, M. F., Stewart, S. E., Eddy, B. E. \& Blackwell, R. H. (1959). Oncogenic effect of tissue-culture preparations of polyoma virus on fetal mice. J. nat. Cancer Inst. 23,1441 .

Stoker, M. \& MacPherson, I. (1961). Studies on transformation of hamster cells by polyoma virus in vitro. Virology, 14, 359.

VANDEPUTTE, M. (1961). Lésions cérébrales chez le rat induites par le virus polyome. Rev. belge Path. 28, 178.

Vogr, M. \& Dulbecco, R. (1960). Virus-cell interaction with a tumor-producing virus. Proc. nat. Acad. Sci., Wash. 46, 365.

Winocour, E. \& SACHS, L. (1961). Cell-virus interactions with the polyoma virus. Virology, 13, 207.

\section{EXPLANATION OF PLATES}

\section{Plate 1}

Fig. 1. Cryostat microtome section of a primary rat kidney sarcoma induced by polyoma virus $\left(\mathbf{R K S}_{2}\right)$. Absence of detectable polyoma antigen by the indirect fluorescence method $(\times 80)$.

Fig. 2. Rat kidney sarcoma (RKS $)_{1}$ grown in tissue culture (day 4). Positive nuclear fluorescence indicating the presence of polyoma antigen $(\times 400)$.

Fig. 3. Rat kidney sarcoma $\left(\mathbf{R K S}_{4}\right)$ grown in tissue culture $(\mathbf{2 4} \mathrm{hr}$.$) . Positive nuclear fluorescence$ $(\times 400)$.

\section{Plate 2}

Fig. 4. Rat subcutaneous fibrosarcoma $\left(\mathrm{RFS}_{1}\right)$ grown in tissúe culture (day 4). Positive cytoplasmic fluorescence $(\times 400)$.

Fig. 5. Rat kidney sarcoma $\left(\mathrm{RKS}_{7}\right)$ grown in tissue culture (day 8). Absence of positive fluorescence $(\times 400)$. 